\title{
PERTURBATIONS OF SMALL MOONS ORBITS DUE TO THEIR \\ ROTATION: THE MODEL PROBLEM
}

\author{
K.GOŹDZIEWSKI and A. J.MACIEJEWSKI \\ Toruń Centre for Astronomy. N. Copernicus University, Poland
}

We consider here the spin-orbit coupling influence on the relative orbital motion of two bodies interacting gravitationally. We assume that one of the bodies is spherically symmetric and the other possesses a plane of dynamical symmetry. In the full non-linear settings, this problem permits coplanar motion when the mass center of the spherically symmetric body moves in the plane. We used this simple model for a qualitative estimation of the changes of the relative orbit in two cases: A) the Sun-asteroid case (the fast rotating rigid body), B) a small satellite of a big planet in resonant rotation.

The motion is described in the rigid body fixed frame. An appropriate change of physical units (Goździewski,1998a) leads to nondimensional dynamical variables and parameters. After that the Hamiltonian of the problem, written in polar variables, is the following

$$
\begin{aligned}
H= & \frac{1}{2}\left(p_{r}^{2}+\frac{p_{\phi}^{2}}{r^{2}}\right)-\frac{1}{r}+\frac{1}{2 I_{3}}\left(G_{3}-p_{\phi}\right)^{2} \\
& -\frac{\epsilon}{2 r^{3}}\left[I_{1}-2 I_{2}+I_{3}-3\left(I_{1}-I_{2}\right) \cos ^{2} \phi\right],
\end{aligned}
$$

where $\left(I_{1}, I_{2}, I_{3}\right)$ are the principal moments of inertia, $(r, \phi)$ are the relative polar coordinates of the point mass in the body frame, $\left(p_{r}, p_{\phi}\right)$ are the canonical momenta, $G_{3}$ represents the constant of total angular momentum, $\epsilon=\left(r_{0} / r\right)^{2}$, and $r_{0}$ is the mean radius of the body.

In the case of model $A$ the disturbing part of the potential may be averaged with respect to the fast variable $\phi$. This gives rise to the perturbed Kepler problem with perturbing potential of the form $\tilde{V}(r)=-\alpha / r^{3}, \alpha>0$. Thus, as a result we obtain the well known effect of the pericenter precession, however for a typical asteroid it is negligible. The semi-major axis and the eccentricity do not change secularly.

In the resonant case the orbit does not exhibit evolutional changes either. The theory describing this situation was developed by Barkin (1975). Assuming the spherical harmonics expansion of the gravitational potential Barkin wrote out the equations of motion averaged through the Delaunay scheme. He showed that the osculating semi-major axis and the eccentricity change periodically in time.

Our aim was to look closely at the short time behaviour of the relative orbit in the resonant case. Here we present an example. For the numerical study we selected Amalthea. As $r_{0} \simeq 100 \mathrm{~km}, r \simeq 1.8 \times 10^{5} \mathrm{~km}$, the small parameter is of the order $\simeq 10^{-7}$. In the case of the 'real' eccentricity $e \simeq 0.003$, the semi-major axis varies periodically with time. This is shown in the upper part of Fig. 1. Its right 

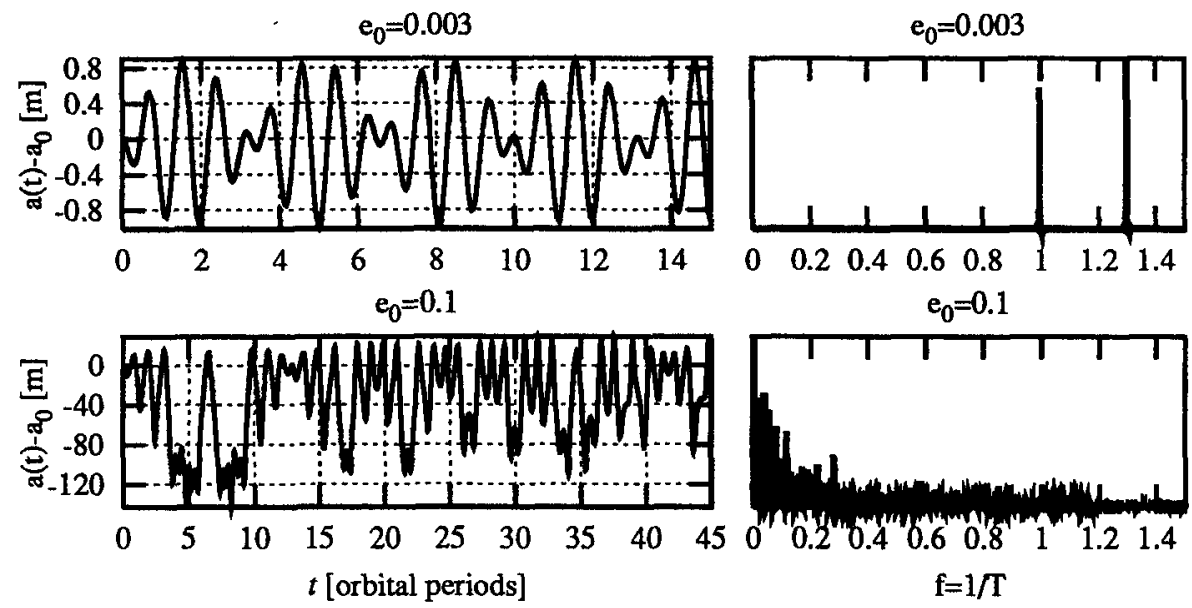

Fig. 1. Short time variation of the osculating semi-major axis of Amalthea in the case B (left panels) and Lomb periodograms of the changes (right panels).

panel shows the Lomb periodogram of the variation. Two dominant frequencies are clearly visible. One of them, equal to 1 , is easily identified with the orbital frequency, the other one is almost the same as the librational, longitudal frequency of the moon (Goździewski, 1998b). The increase of the initial eccentricity up to 0.1 leads to an unexpected, interesting result: the osculating semi-major axis varies chaotically. The corresponding Lomb periodogram shows that the spectrum is almost continuous with maximum of power near the zero frequency. Let us note very large amplitude of variations, of the order of $200 \mathrm{~m}$. The scale of this effect justifies that it is worth detailed study.

This work was supported by N. Copernicus Grant No. 315-A.

\section{References}

Barkin, Y.W. (1952) Intermediate plane motion of a rigid body in the gravitational field of a sphere. Astron. Zh., 52(5), pp. 1076-1083.

Goździewski K., and Maciejewski, A.J. (1998a) Equations of motion and Lagrangian equilibria in the special version of the three body problem, in preparation.

Goździewski K., and Maciejewski, A.J. (1998b) Semi-analytical model of libration of a rigid moon orbiting an oblate planet. Astron. Astroph., (in print). 